Original article

Received: 8 April 2016 / Accepted: 26 September 2017

\title{
CONCEPT OF AHRS ALGORITHM DESIGNED FOR PLATFORM INDEPENDENT IMU ATTITUDE ALIGNMENT
}

\author{
Dariusz Tomaszewski ${ }^{1}$, Jacek Rapiński ${ }^{1}$, Renata Pelc-Mieczkowska ${ }^{2}$ \\ ${ }^{1}$ Institute of Geodesy, \\ The Faculty of Geodesy, Geospatial and Civil Engineering, \\ University of Warmia and Mazury in Olsztyn \\ ${ }^{2}$ Institute of Geoinformation and Cartography, \\ The Faculty of Geodesy, Geospatial and Civil Engineering, \\ University of Warmia and Mazury in Olsztyn
}

\begin{abstract}
Nowadays, along with the advancement of technology one can notice the rapid development of various types of navigation systems. So far the most popular satellite navigation, is now supported by positioning results calculated with use of other measurement system. The method and manner of integration will depend directly on the destination of system being developed. To increase the frequency of readings and improve the operation of outdoor navigation systems, one will support satellite navigation systems (GPS, GLONASS ect.) with inertial navigation. Such method of navigation consists of several steps. The first stage is the determination of initial orientation of inertial measurement unit, called INS alignment. During this process, on the basis of acceleration and the angular velocity readings, values of Euler angles (pitch, roll, yaw) are calculated allowing for unambiguous orientation of the sensor coordinate system relative to external coordinate system. The following study presents the concept of AHRS (Attitude and heading reference system) algorithm, allowing to define the Euler angles. The study were conducted with the use of readings from low-cost MEMS cell phone sensors. Subsequently the results of the study were analyzed to determine the accuracy of featured algorithm. On the basis of performed experiments the legitimacy of developed algorithm was stated.
\end{abstract}

Keywords: Attitude Alignment, AHRS, Integrated Navigation, GPS/IMU

\section{Introduction}

In can be noticed that the use of microelectronic sensors help people in everyday life. Micro systems are used in a variety of fields to increase productivity, improve functionality and reduce their operating costs. Attitude heading reference system (AHRS) build on MEMS 


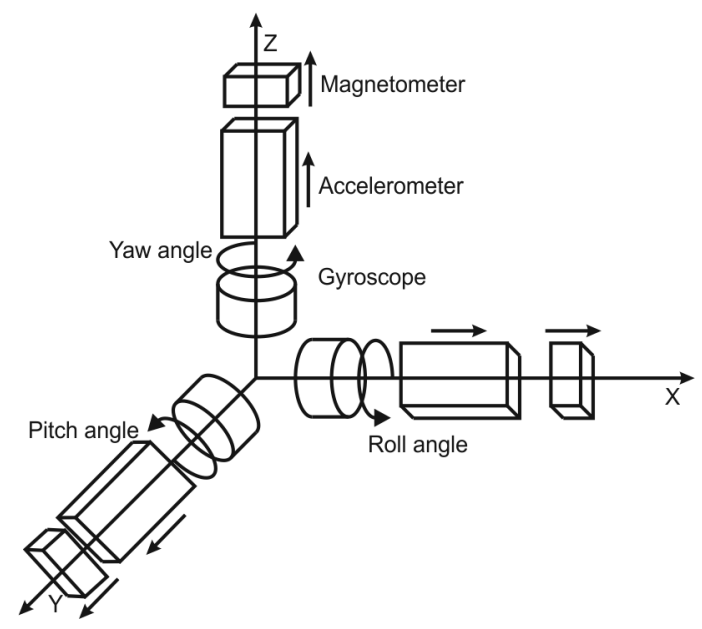

Fig. 1. The inertial measurement unit body coordinate system.

IMU (Inertial Measurement Unit) is one of mentioned features used in applications such as underwater navigation (Emami \& Taban, 2017), motion capture (Sasani, 2016), augmented reality (Yang Ling, 2016) and aircraft guidance (Tang Daquan, 2016). As well as a support for obtaining the initial orientation of IMU for the purpose of IMU/GPS integrated navigation. The following stage of navigation is also called INS attitude alignment and it is the first part of IMU/GPS integrated navigation. Such type navigation is one of the most commonly used method of increasing the frequency and integrity of satellite navigation (GPS, GLONASS ect.) (Li et al., 2016). The band sensors implemented within the IMU allows for position, velocity and attitude determination on the basis of readings carried out without the involvement of external signals (Woodman, 2007). At the same time, due to the relatively high noise and the accumulation of errors, the MEMS - based inertial navigation cannot be used as a standalone navigation system.

AHRS system is build on a basis of IMU unit with appropriate algorithm implemented. During this process, on the basis of acceleration and the angular velocity readings, values of Euler angles (pitch, roll, yaw) are calculated allowing for unambiguous orientation of the sensor coordinate system relative to external coordinate system (Sabet et al., 2017). After determination of these values the proper inertial navigation begins. The inertial measurement unit usually consists of three components : three axial accelerometer, three axial gyroscope and three axial magnetometer Figure 1 (Farrel, 2008).

Construction of the IMU module ensures the measurements of linear acceleration, angular velocity and the induction of earth magnetic field in three mutually orthogonal direction. The axes off all sensors are parallel, shearing the same origin. Such solution of the sensors seating defines the IMU body coordinate system called the body frame.

The first stage of inertial navigation is the attitude alignment. In these step the orientation of the IMU body with respect to an external coordinate system (eg. WGS 84) is determined. Through orientation one will understand the angles between the axes of body frame and the axes external coordinate system. These values are the Euler angles called pitch, roll and yaw (Figure 2); (Foxlin, 2005). Their precise determination allows to eliminate the influence of gravity acceleration and Corriolis force from subsequent navigation. Traditionally the most commonly used method for Euler angles determination is performing attitude computation on the basis of mean sensor readings made within few minutes during which the IMU module remained stationary. These solution is sufficient however, it occupies a lot of time. Moreover, often even a slight influence on the sensor system during this process can 

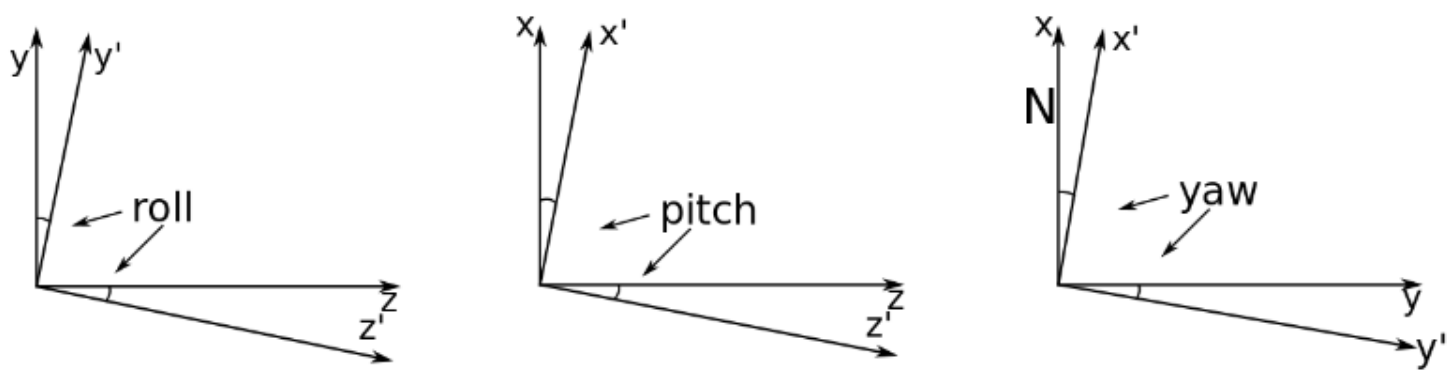

Fig. 2. Presentation of the Euler angles.

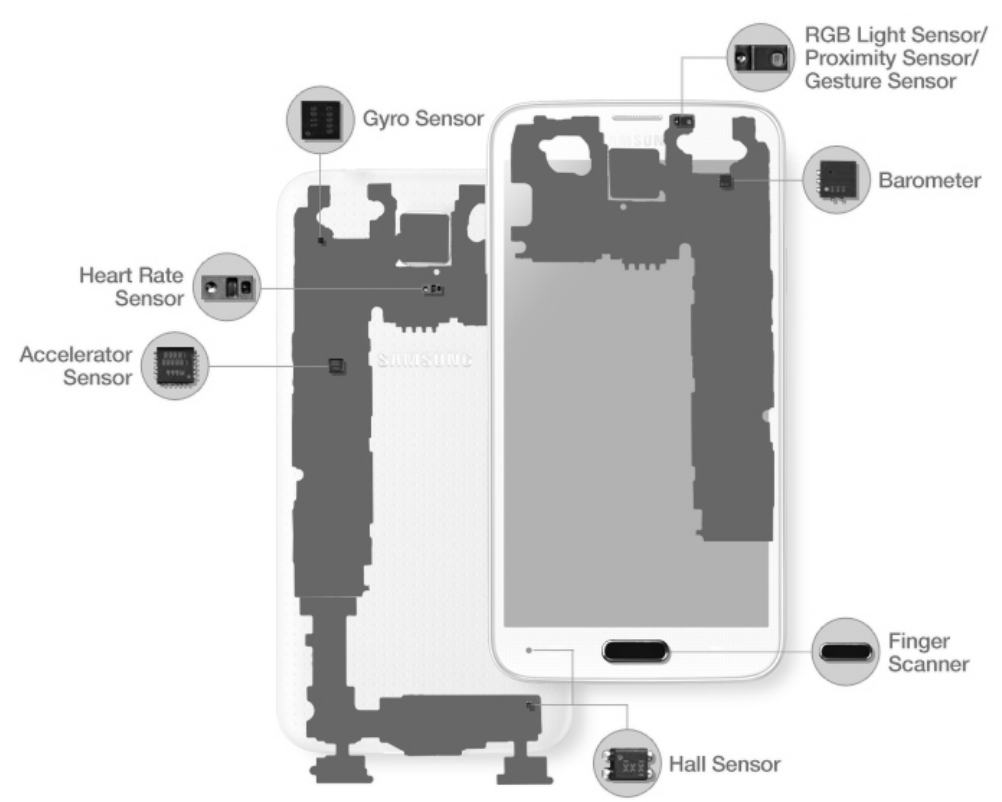

Fig. 3. Presentation of the Euler angles.

cause irregularities in the calculation. As a consequence of these irregularities the incorrect determination of the initial IMU orientation can be noticed. This will have a significant impact on the results of further navigation. The use of AHRS algorithm for sensor orientation determination eliminates these two factors. The computation is based on Kalman filter which ensures the calculation of searched quantities from two sources. This solution reduces the time length of the process from few minutes to few seconds. At the same time unexpected impact on the sensors, during the alignment, does not change the accuracy of conducted calculation.

The following study presents the concept of AHRS (Attitude and heading reference system) algorithm, allowing to define the Euler angles for IMU attitude alignment. Subsequently the results of algorithm functionality test is presented. The study were conducted with the use of readings from low-cost MEMS sensors installed within Samsung galaxy S3 and Samsung galaxy S5 cell phones (Figure 3 ). Proposed algorithm can be also used for self-build platforms as it was described in (Tomaszewski, Rapińki, \& Śmieja, 2015). The results of conducted research were analyzed in order to determine the accuracy of calculations made by developed algorithm. 


\section{Attitude Alignment}

As it was mentioned in the introduction the attitude alignment is the first stage of INS/GPS integrated navigation system. In this step the IMU module remains stationary while collecting observational data from accelerometers, gyroscopes and magnetometer. Data gathered form accelerometer illustrate the effect of gravity on all three axes. On the basis of these readings one can determine the values of pitch end roll angles. These calculations are performed with assumption that the perfectly horizontal device would have linear acceleration readings only in vertical direction ( $z$ axis). If that condition is not satisfied the inclination (pitch and roll) of the IMU is calculated with the use of following formula (Groves, 2008)(Cui et al., 2017).

$$
\begin{gathered}
\theta=\tan ^{-1}\left(\frac{-a_{x}}{\left.\sqrt{1\left(a_{x}^{2}\right)+a_{z}^{2}}\right)}\right) \\
\phi=\tan ^{-1}\left(\frac{-a_{x}}{a_{z}}\right)
\end{gathered}
$$

Where:

$a_{x}, a_{y}, a_{z}$ - measurements made by respective axes of accelerometer. Usually the mean readings gathered during few minutes of observations

$\theta, \phi$ - pitch and roll angles respectively

Determination the yaw angle value will depend directly on the class of the device used in considered measurement unit. If high class device is used, the yaw angle can be calculated on the basis of earth rotation measurement made by the stationary gyroscope. Then the result is obtained with the use of following formula (Tomaszewski, Rapiński, \& Śmieja, 2015) :

$$
\psi=\tan ^{-1}\left[\frac{\omega_{x} \cos \phi+\omega_{z} \sin \phi}{\omega_{y} \cos \theta+\omega_{x} \sin \theta \sin \phi-\omega_{z} \cos \phi \sin \theta}\right]
$$

Where:

$\omega_{x}, \omega_{y}, \omega_{z}$ - measurements of angular velocity made by around axes of gyroscope $\theta, \phi, \psi$ - pitch, roll and yaw angles respectively

This process is called gyrocompassing. However, its use is significantly limited, because it requires a device that is able to detect the angular velocity less then about $7 * 10^{-5} \mathrm{rad} / \mathrm{s}$. This means that the gyroscope would have to characterize the ARW (Angular random Walk) error less then $5^{\circ} / h$. Only expensive devices have such accuracy. IMU mounted inside of the mobile phone do not meet this requirement (Tomaszewski, Rapiński, \& Śmieja, 2015). Due to that fact the determination of yaw angle in case of mobile devices is made on the basis of magnetic induction readings performed by magnetic field sensor called magnetometer. Yaw angle calculation with the use of magnetometer is more problematic because this device requires calibration before start of every navigation. This calibration is performed to create a distribution map of magnetic induction around the device affected by intrinsic zero field offset and permanent magnetic fields within PCB generated by magnetized ferromagnetic materials (Henriksson, 2013). These influence is called Hard-Iron offset and is the cause of jamming the yaw angle calculations with the use of magnetometer. In order to eliminate this effect the following algorithm is usually used:

Considered device is rotated over three axes to create the map of raw measurements distribution. Obtained values satisfies following equation (Ozyagcilar, 2012): 


$$
\left(B_{P}-V\right)^{T}\left(B_{P}-V\right)=B^{2}
$$

Where:

$B$ - is the magnitude of geomagnetic sphere.

$B_{P}-\left|\begin{array}{c}B_{P x} \\ B_{P y} \\ B_{P z}\end{array}\right|$ - vector of magnetometer output in the absence of Hard-Iron offset.

$V-\left|\begin{array}{l}V_{x} \\ V_{y} \\ V_{z}\end{array}\right|$ the Hard-Iron offset vector.

The residual errors $r[i]$ in $\mathrm{i}$-th is presented by a following equation.

$$
r(i)=\left|B_{p x}(i)^{2}+B_{p y}(i)^{2}+B_{p z}(i)^{2}\right|-\left|\begin{array}{c}
B_{P x(i)} \\
B_{P y}(i) \\
B_{P z}(i) \\
1
\end{array}\right|^{T}\left|\begin{array}{c}
2 V_{x} \\
2 V_{y} \\
2 V_{z} \\
B^{2}-V_{x}^{2}-V_{y}^{2}-V_{z}^{2}
\end{array}\right|
$$

If one will consider the $\mathrm{n}$ number of observations in the map of raw measurements that will be used for computation of the Hard-Iron offset. The formula would look as follows://

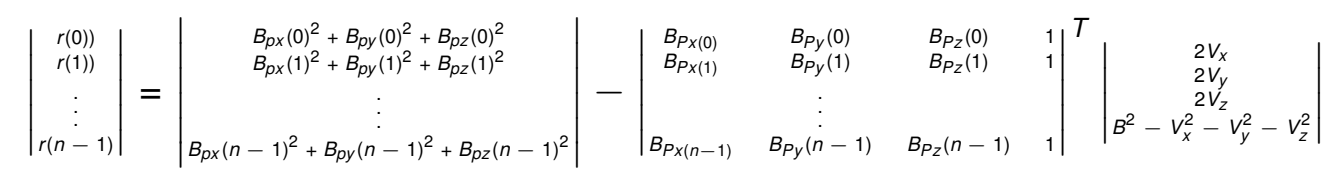

Equation 6 can be abstracted to:

$$
r=Y-X \beta
$$

Where:

$Y$ - vector of known independent variables

$X$ - matrix of known magnetometer measurements

$\beta$-solution vector from each the four Hard-Iron offset parameters

$r$ - vector of error residuals

These considerations leads to the minimization of performance function $P$ with the use of the least squares method. The $P$ function is as follows:

$$
P=r(0)^{2}+r(1)^{2}+\ldots+r(n-1)^{2}=(Y-X \beta)^{T}(Y-X \beta)
$$

The solution of equation 8 is:

$$
\beta=\left(X^{T} X\right)^{-1} X^{T} Y
$$

The minimized performance function will give the values of $V x, V y$, and $V z$ as well as the value of magnitude of geomagnetic sphere $B$. Once all the quantities are computed the value of yaw angle can be calculated with the use of formula 10 (Freescale, 2012).

$$
\psi=\tan ^{-1}\left[\frac{\left(B_{p z}-V_{z}\right) \sin \phi-\left(B_{p y}-V_{y}\right) \sin \phi}{\left(B_{p x}-V_{x}\right) \sin \theta+\left(B_{p y}-V_{y}\right) \sin \theta \sin \phi+\left(B_{p z}-V_{z}\right) \cos \phi \sin \theta}\right]
$$




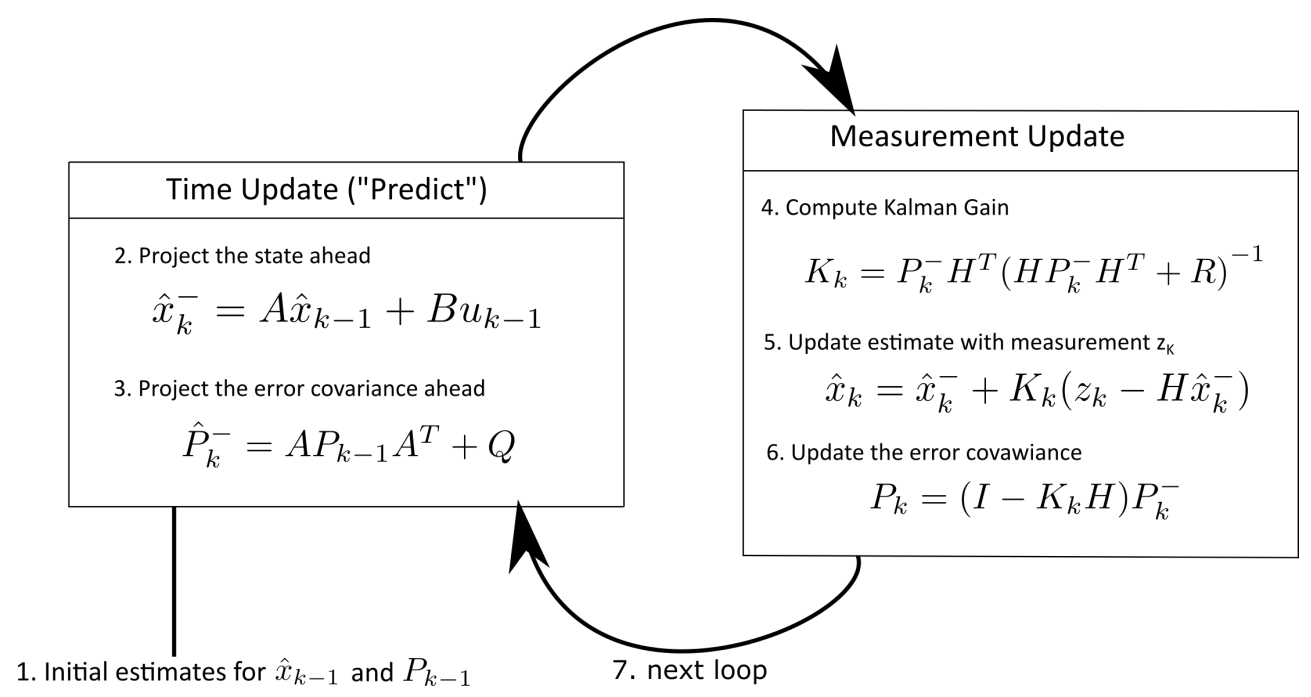

Fig. 4. The principle of Kalman filter.

Using the equations 1,2 and 3 or 10 one is capable of defining the initial orientation of IMU in space. However, usually this process provides the pitch, roll and yaw angle with the use of only one sensor without any external aid. Calculated quantities area subject to an errors directly dependent on a accuracy of used sensor. In addition the process is time-consuming due to the fact that the IMU must remain stationary by specific period of time to obtain desired accuracy. The authors wish to introduce, based on Kalman filter, AHRS algorithm that provides acceleration of calculations, while maintaining sufficient accuracy (Yadav \& Bleakley, 2014).

\section{Concept of Kalman filter}

For the purpose of the AHRS algorithm the Kalman filter is used. This is a sequential recursive algorithm that provides an optimal least mean variance estimation of the searched states. In the case of AHRS these states would be the values of Euler angles. In addition it provides the information about statistical accuracy of each calculated state. The main advantage of the Kalman filter is that it allows combining measurements from different sources. Then on the basis of known accuracy it calculates the weights and determine the impact of each observable on the final result. KF is a recursive algorithm which estimates the states of the system by operating as a feedback loop. On the basis of designed system model the filter calculates the state at known epoch $t$. These state is compared with measurement system result producing the optimal filter output. A KF comprises five equations which operate in a sequential manner. The flow of algorithm is based on two states: prediction state and correction state (Noureldin et al., 2013). The idea is depicted in figure 4.

The steps of the process are explained below (Simončič et al., 2015):

1. First step is the initialization of the filter. The filter is provided in some initial state $\hat{x}_{0}$ and the initial covariance matrix $P_{0}$. The initial values of noise covariance matrix $Q$ and measurement covariance matrix $R$ are also provided in these step. The values of these components are usually chosen on the basis of values estimated during previous usage of the filter. 
2. In the second filter step is the first part of prediction process. Then on a basis of created state transition matrix $A$ the initial state is propagated from epoch $t-1$ to epoch $t$. Which gives the predicted state $\hat{x}_{t}(-)$.

3. The third step is the prediction of covariance of predicted state $P_{t}$. These part is calculated on the basis of known previous state covariance matrix $P_{(t-1)}$, process noise covariance $Q_{(t-1)}$. Sometimes also the noise distribution matrix $G_{(t-1)}$ is added.

4. In forth step the value of Kalman gain $K_{t}$ is calculated to weight the predicted state and calculate what impact will the predicted values have on final filter state.

5. In the fifth part the predicted state is corrected whenever the measurement is received. In these step on the basis of known difference between the predicted measurement $H \hat{x}_{t}(-)$ and the actual measurement $z_{t}$, the corrected state $\hat{x}_{t}(+)$ is estimated. The impact of the prediction is adjusted on the basis of known Kalman gain $K_{t}$.

6. Now on the basis of known Kalman gain $K_{t}$ the value of error covariance is recalculated to state $P_{t}(+)$ to indicate the level of trust in the corrected estimate $\hat{x}_{t}(+)$.

7. Now the Kalman Filter is ready to start next loop from step 2.

\section{AHRS Algorithm}

AHRS is an algorithm that allows to define an inner orientation of the device. Its main advantage over traditional attitude determination (with the use of accelerometer sensors only) is the use of hole set of IMU unit sensors for Euler angles determination. To integrate data from accelerometer, gyroscope and magnetometer the Kalman filter algorithm was used. This kind of filtration allows to mitigate errors that affects the calculation results conducted with the use of raw observational data (Wang et al., 2015). Small errors gyroscope readings allows one to reduce calculation errors caused by high accelerometer noise. At the same time the stability pitch, roll and yaw calculation conducted on the basis of accelerometer and magnetometer readings allows to mitigate gyroscope drift impact on the final results. The design of filter matrices is presented below.

State vector $\hat{x}_{t}(-)$ consists of Euler angles pitch, roll and yaw $(\theta, \phi, \psi)$ and drifts of the gyroscope corresponding to particular angle $(\mathrm{d} \theta, \mathrm{d} \phi, \mathrm{d} \psi)$. Initial drifts are calculated on the basis of first few measurements of stationary gyro (less than a second of observation). Initial values of the pitch, roll and yaw angles can be computed with raw accelerometer and magnetometer measurements or can be assumed to be 0 and in first yew steps of calculation the filter will calculate appropriate value.

$$
\hat{x}_{t}(-)=\left|\begin{array}{c}
\theta \\
\phi \\
\psi \\
d \theta \\
d \phi \\
d \psi
\end{array}\right|
$$

State transition matrix A ensures the angle from previous Kalman estimation and removes impact of the drift from the angle prediction. 


$$
A=\left|\begin{array}{cccccc}
1 & 0 & 0 & -d t & 0 & 0 \\
0 & 1 & 0 & 0 & -d t & 0 \\
0 & 0 & 1 & 0 & 0 & -d t \\
0 & 0 & 0 & 1 & 0 & 0 \\
0 & 0 & 0 & 0 & 1 & 0 \\
0 & 0 & 0 & 0 & 0 & 1
\end{array}\right|
$$

$B$ is the distribution matrix with allows for multiplication of gyroscope measurements by the time difference from the previous measurement.

$$
B=\left|\begin{array}{ccc}
d t & 0 & 0 \\
0 & d t & 0 \\
0 & 0 & d t \\
0 & 0 & 0 \\
0 & 0 & 0 \\
0 & 0 & 0
\end{array}\right|
$$

$u_{(t-1)}$ is the prediction input. This solution is used due to the fact that there is more than one system that is able to determine the values of searched angles. In these vector there are raw gyroscope readings ( $g x, g y, g z)$ used as an update in prediction state of the filter .

$$
u_{(t-1)}=\left|\begin{array}{l}
-g y \\
-g x \\
-g z
\end{array}\right|
$$

Initial covariance matrix and covariance matrix of predicted state are the same. On the diagonal the covariance of pitch, roll, yaw and covariance of the drifts are placed.

$$
P_{0}=Q=\left|\begin{array}{cccccc}
m \theta & 0 & 0 & 0 & 0 & 0 \\
0 & m \phi & 0 & 0 & 0 & 0 \\
0 & 0 & m \psi & 0 & 0 & 0 \\
0 & 0 & 0 & m d \theta & 0 & 0 \\
0 & 0 & 0 & 0 & m d \phi & 0 \\
0 & 0 & 0 & 0 & 0 & m d \psi
\end{array}\right|
$$

In measurement vector the values of Euler angles were placed. Pitch and roll taken for these part of calculation are determined with the use of accelerometer readings $\left(a_{x}\right.$, $\left.a_{y}, a_{z}\right)$. Yaw angle is estimated on the basis of calibrated magnetometer readings $\left(B_{p x}\right.$, $\left.B_{p y}, B_{p z}\right)$ expunged for the components of Hard-Iron offset $\left(V_{x}, V_{y}, V_{z}\right)$ calculated during magnetometer calibration.

$$
Z_{t}=\left|\begin{array}{c}
\tan ^{-1}\left(\frac{-a_{x}}{\sqrt{a_{x}^{2}+a_{z}^{2}}}\right) \\
\tan ^{-1}\left(\frac{-a_{x}}{a_{z}}\right) \\
\tan ^{-1}\left[\frac{\left(B_{p z}-V_{z}\right) \sin \phi-\left(B_{p y}-V_{y}\right) \sin \phi}{\left(B_{p x}-V_{x}\right) \sin \theta+\left(B_{p y}-V_{y}\right) \sin \theta \sin \phi+\left(B_{p z}-V_{z}\right) \cos \phi \sin \theta}\right]
\end{array}\right|
$$

In the measurement vector there are only Euler angles since the drifts are not obtained from second measurement system. Due to the fact that measured values are synonymous 


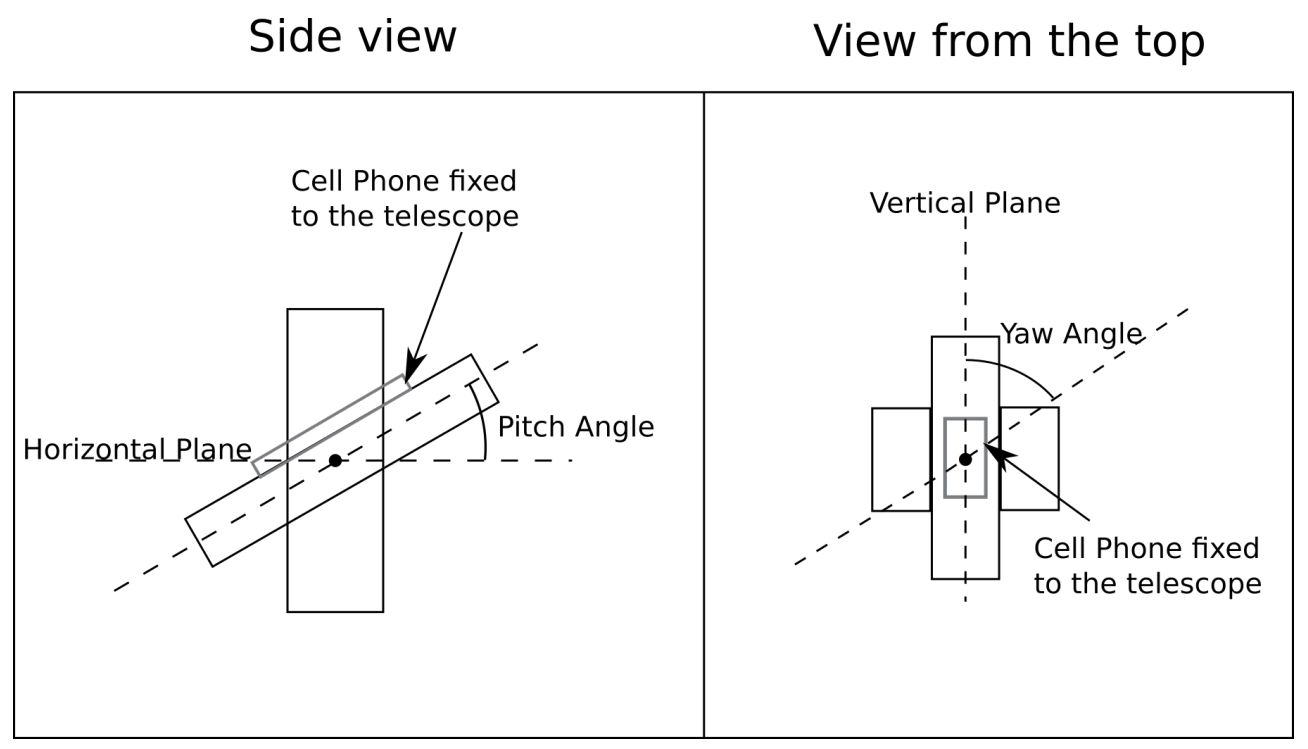

Fig. 5. Installation of cell phone installation during the experiment.

to the ones that are predicted, the design matrix $\mathrm{H}$ have the following form:

$$
H=\left|\begin{array}{llllll}
1 & 0 & 0 & 0 & 0 & 0 \\
0 & 1 & 0 & 0 & 0 & 0 \\
0 & 0 & 1 & 0 & 0 & 0
\end{array}\right|
$$

the measurement covariance matrix is diagonal with covariance of each calculated angle on the diagonal.

$$
H=\left|\begin{array}{ccc}
m_{z} \theta & 0 & 0 \\
0 & m_{z} \phi & 0 \\
0 & 0 & m_{z} \psi
\end{array}\right|
$$

\section{The test of attitude alignment accuracy conducted with the use of designed algo- rithm}

The study of designed algorithm was made with the use of sensor readings from too cell phones, Samsung Galaxy S3 and Samsung Galaxy S5. To check the correctness of estimated results three tests were made. In the first test the cell phones reminded stable at nearly horizontal plane. In the second test the phones was turned over $y$ axis with 30 degrees. In the last test the value of yaw angle was changed. To ensure the accuracy of performed rotations the cell phones were fixed to the telescope of TS30 Leica total station (Figure. 5).

The first tested device was Samsung galaxy S3. On the following graphs the results of Euler angle calculation's are depicted (Figure 6, 7, 8). During each of considered experiments the pitch, roll and yaw angle were calculated to check the impact of attitude changes on all estimated values. The black color was used to depict the AHRS result. On the other hand grey marcs results of one-epoch calculation conducted on the basis of accelerometer (pitch and roll) and magnetometer (yaw) readings. 

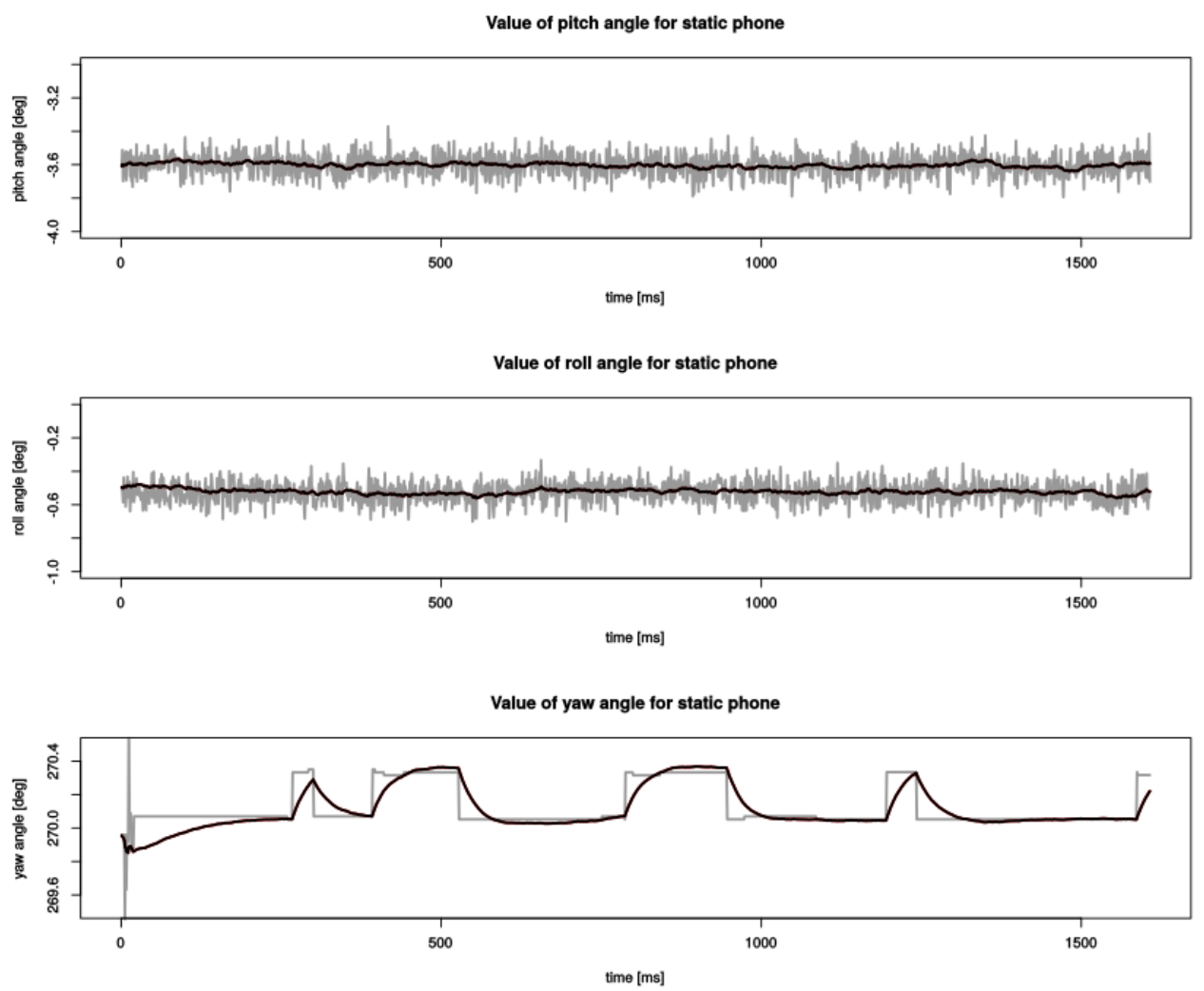

Fig. 6. Values of Euler angles calculated with statically situated Galaxy S3 cell phone.
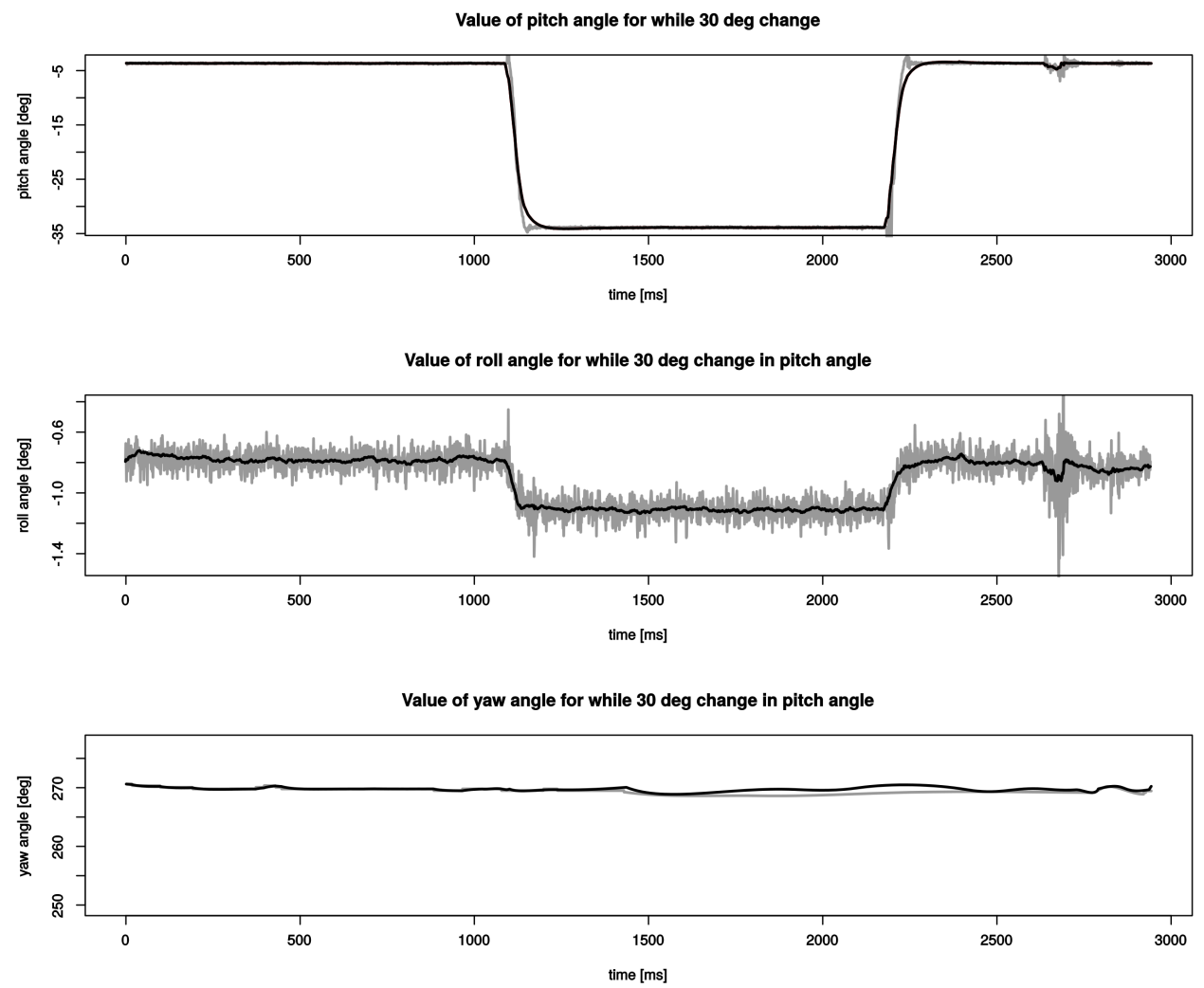

Fig. 7. Values of Euler angles calculated during the 30 deg change in pitch angle (Galaxy S3 cell phone). 

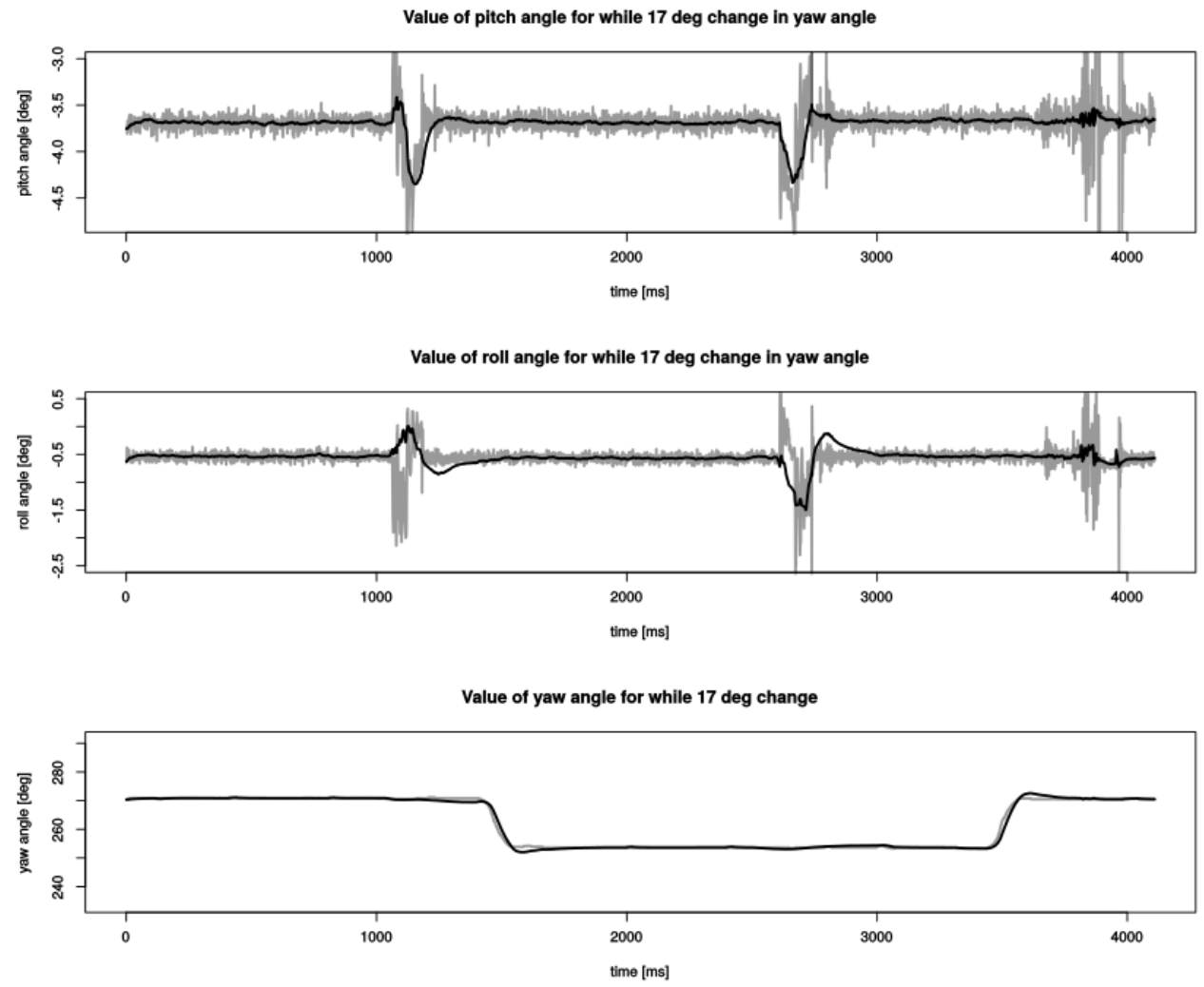

Fig. 8. Values of Euler angles calculated during the 17 deg change in yaw angle (Galaxy S3 cell phone).

Some statistics of conducted calculations are shown in the table below (Table 1).

Tab. 1. Statistics of calculations conducted with Galaxy S3 cell phone.

\begin{tabular}{|c|c|c|c|c|c|c|}
\hline & Min Pitch [deg] & Max Pitch [deg] & $\begin{array}{c}\text { Static Phone } \\
\text { Mean Pitch [deg] }\end{array}$ & Pitch Median [deg] & Pitch Standard Deviation & Pitch Variance \\
\hline AHRS & -3.639 & 3.567 & -3.602 & -3.601 & 0.013 & 0.0002 \\
\hline \multirow[t]{2}{*}{ Raw Data } & -3.794 & 3.371 & -3.602 & -3.602 & 0.063 & 0.004 \\
\hline & $\begin{array}{c}\text { Min Pitch } \\
\text { Change [deg] }\end{array}$ & $\begin{array}{c}\text { Max Pitch } \\
\text { Change [deg] }\end{array}$ & $\begin{array}{c}30 \text { deg change in Pitch } \\
\text { Mean Pitch } \\
\text { Change [deg] }\end{array}$ & $\begin{array}{l}\text { Pitch Change } \\
\text { Median [deg] }\end{array}$ & $\begin{array}{c}\text { Pitch Change } \\
\text { Standard Deviation }\end{array}$ & $\begin{array}{l}\text { Pitch Change } \\
\text { Variance }\end{array}$ \\
\hline AHRS & 30.182 & 30.285 & 30.236 & 30.239 & 0.018 & 0.0003 \\
\hline \multirow[t]{2}{*}{ Raw Data } & 29.940 & 30.512 & 30.245 & 30.225 & 0.104 & 0.0107 \\
\hline & $\begin{array}{c}\text { Min Yaw } \\
\text { Change [deg] }\end{array}$ & $\begin{array}{c}\text { Max Yaw } \\
\text { Change [deg] }\end{array}$ & $\begin{array}{c}17 \text { deg change in yaw } \\
\text { Mean Yaw } \\
\text { Change [deg] }\end{array}$ & $\begin{array}{l}\text { Yaw Change } \\
\text { Median [deg] }\end{array}$ & $\begin{array}{c}\text { Yaw Change } \\
\text { Standard Deviation }\end{array}$ & $\begin{array}{c}\text { Yaw Change } \\
\text { Variance }\end{array}$ \\
\hline AHRS & 12.748 & 17.464 & 17.018 & 17.230 & 0.603 & 0.363 \\
\hline Raw Data & 12.848 & 17.564 & 17.018 & 17.230 & 0.603 & 0.363 \\
\hline
\end{tabular}

The second study was conducted with the use of Samsung Galaxy S5. The choice of this device was determined by the precision of sensors installed in these models. Galaxy S5 as a lot younger model then Galaxy S3 and due to that fact the MEMS sensors mounted within this device are far more accurate the in older model. Similar tests, as in Samsung Galaxy S3, were performed on this model. Graphs representing the results of the study are presented below (figure 9, 10, 11).

Some statistics of conducted calculations are shown in the table below (Table 2). 


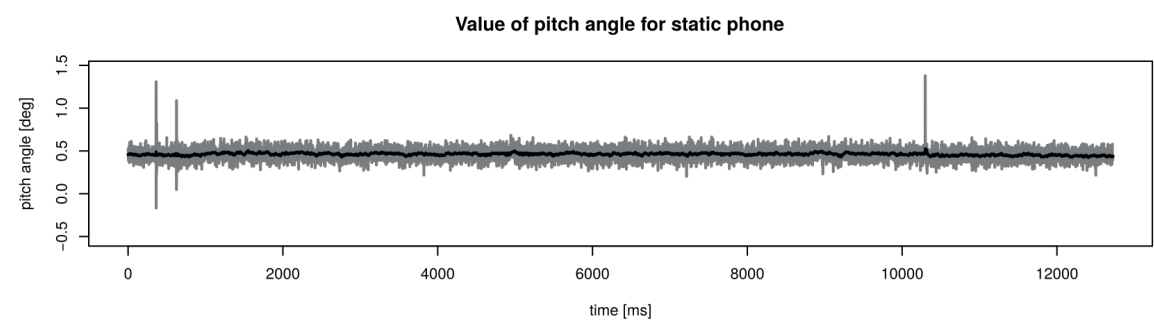

Value of roll angle for static phone

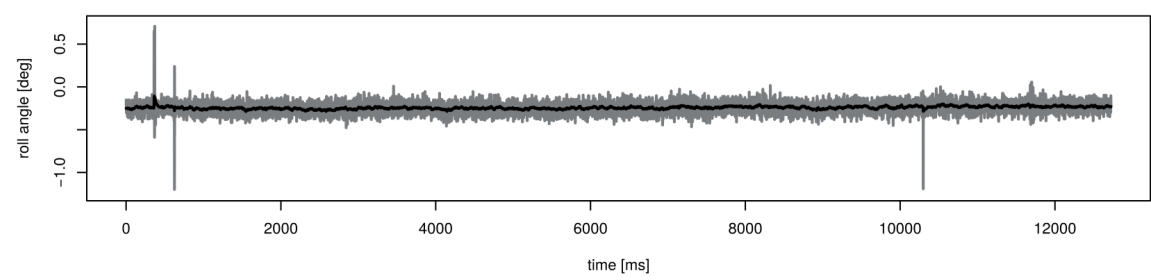

Value of yaw angle for static phone

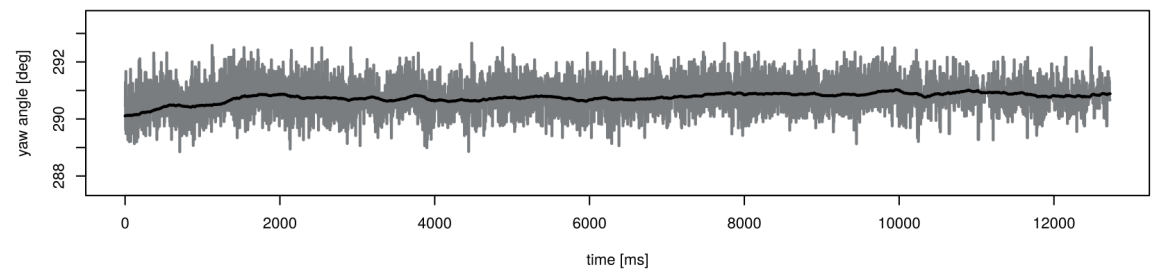

Fig. 9. Values of Euler angles calculated with statically situated Galaxy S5 cell phone.
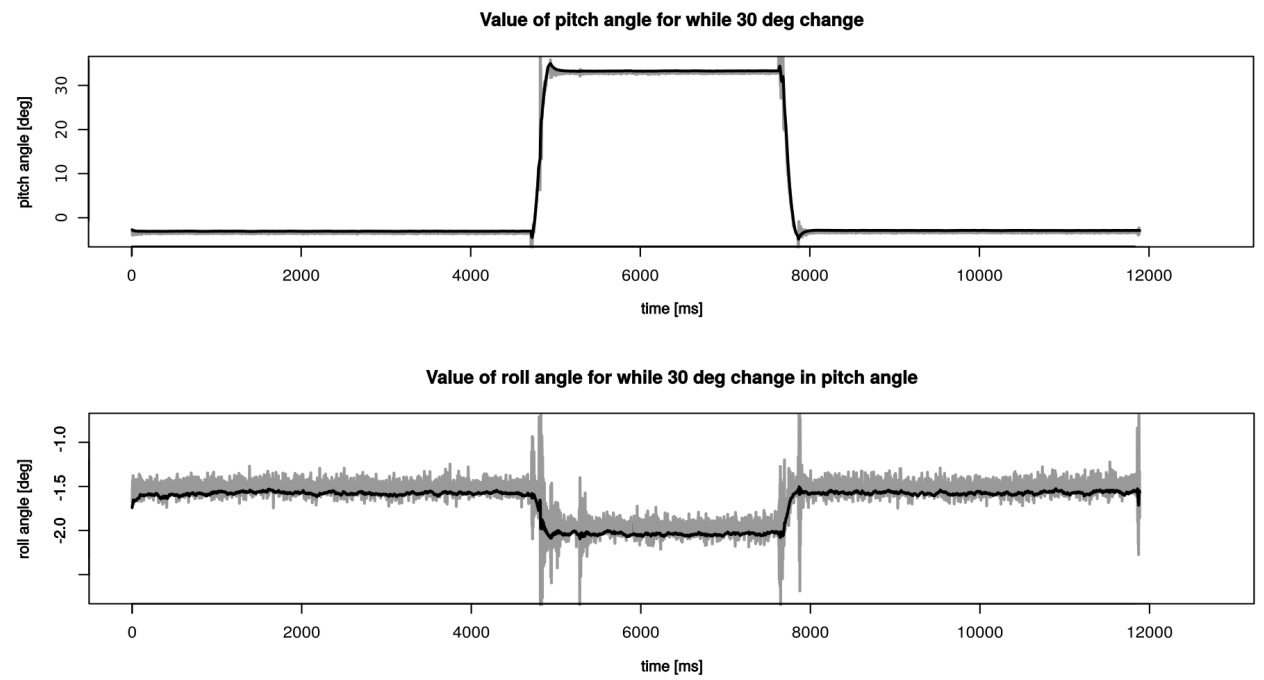

Value of yaw angle for while $30 \mathrm{deg}$ change in pitch angle

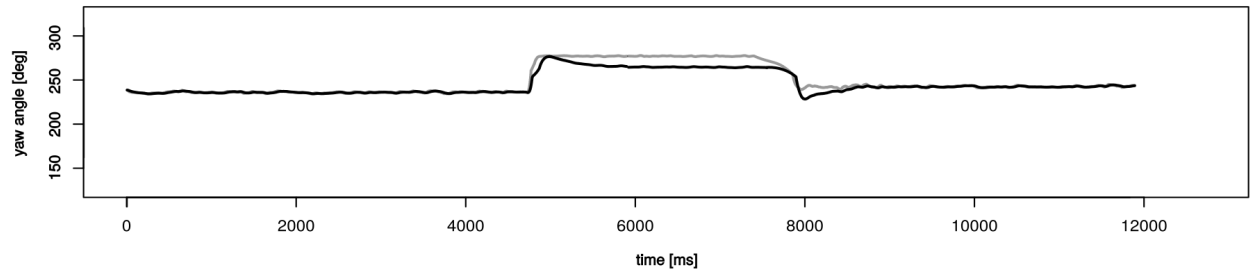

Fig. 10. Values of Euler angles calculated during the 30 deg change in pitch angle (Galaxy S5 cell phone). 

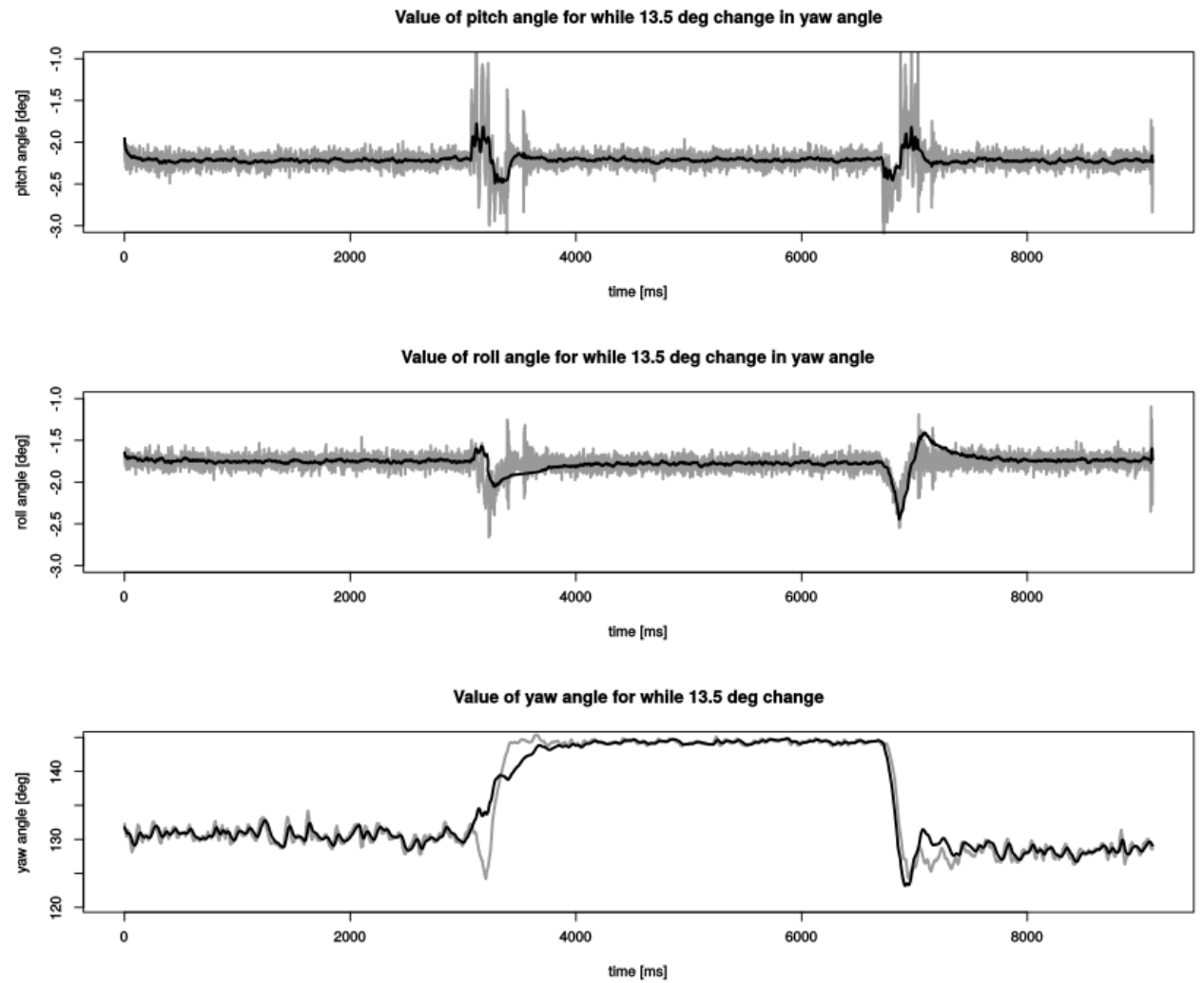

Fig. 11. Values of Euler angles calculated during the 13.5 deg change in yaw angle (Galaxy S5 cell phone).

Tab. 2. Statistics of calculations conducted with Galaxy S5 cell phone.

\begin{tabular}{|c|c|c|c|c|c|c|}
\hline & Min Pitch [deg] & Max Pitch [deg] & $\begin{array}{l}\text { Static Phone } \\
\text { Mean Pitch [deg] }\end{array}$ & Pitch Median [deg] & Pitch Standard Deviation & Pitch Variance \\
\hline AHRS & 0.422 & 0.522 & 0.462 & 0.462 & 0.0129 & 0.0002 \\
\hline \multirow[t]{2}{*}{ Raw Data } & -0.167 & 1.180 & 0.461 & 0.461 & 0.0632 & 0.0040 \\
\hline & $\begin{array}{l}\text { Min Pitch } \\
\text { Change [deg] }\end{array}$ & $\begin{array}{l}\text { Max Pitch } \\
\text { Change [deg] }\end{array}$ & $\begin{array}{c}30 \text { deg change in Pitch } \\
\text { Mean Pitch } \\
\text { Change [deg] }\end{array}$ & $\begin{array}{l}\text { Pitch Change } \\
\text { Median [deg] }\end{array}$ & $\begin{array}{l}\text { Pitch Change } \\
\text { Standard Deviation }\end{array}$ & $\begin{array}{l}\text { Pitch Change } \\
\text { Variance }\end{array}$ \\
\hline AHRS & 29.902 & 30.026 & 29.981 & 29.981 & 0.0214 & 0.0004 \\
\hline \multirow[t]{2}{*}{ Raw Data } & 29.637 & 30.376 & 29.978 & 29.977 & 0.1051 & 0.0110 \\
\hline & $\begin{array}{c}\text { Min Yaw } \\
\text { Change [deg] }\end{array}$ & $\begin{array}{c}\text { Max Yaw } \\
\text { Change [deg] }\end{array}$ & $\begin{array}{c}13.5 \text { deg change in yaw } \\
\text { Mean Yaw } \\
\text { Change [deg] }\end{array}$ & $\begin{array}{l}\text { Yaw Change } \\
\text { Median [deg] }\end{array}$ & $\begin{array}{l}\text { Yaw Change } \\
\text { Standard Deviation }\end{array}$ & $\begin{array}{l}\text { Yaw Change } \\
\text { Variance }\end{array}$ \\
\hline AHRS & -15.624 & -11.391 & -13.669 & -13.939 & 1.000 & 1.001 \\
\hline Raw Data & -16.124 & -9.647 & -13.732 & -13.927 & 1.262 & 1.594 \\
\hline
\end{tabular}

\section{Conclusion}

On the basis of conducted studies the performance of designed AHRS algorithm was determined. The study indicates that tested algorithm works similarly in case of both used cell phones. And its accuracy is closely related to the type off sensors mounted within tested device. A comparison of average values of Euler angles indicates that precision of estimated mean is similar for raw and filtered observational data. For example, the mean pitch value for static Galaxy S5 equals $0.462^{\circ}$ for AHRS values and $0.461^{\circ}$ for row data. However, as can be seen from the presented tables, standard deviation and variance values for filtered data are much lower than in case of raw data. Standard deviation is $0.0129^{\circ}$ and variance is 0.0002 in the case of static Galaxy S5 AHRS estimation. On the other hand, in the same case, standard deviation for raw data is $0.0632^{\circ}$ and variance is 0.0040 . Similar dependencies 
can also be observed in the case of other tests. Practically in all estimations of pitch and roll angles the values of standard deviation are four times lower for AHRS algorithm and at least twenty times lower in case of variance. This suggests that single estimation has higher accuracy for AHRS algorithm. The following statement can be also confirmed after analysis of above graphs with clearly shows a significant smoothing in gathered data. It can be noticed that for yaw calculations the statistics comes out differently. For Galaxy S3 cell phone it is related with very low accuracy of magnetometer. In this case the results comes out with similar accuracy. As for the Galaxy S5 the difference in accuracy of yaw angle calculation is not as large as for pitch and roll estimations. Presented algorithm has some limitations that has to be mentioned. In special circumstances it can be exposed to gimbal lock which will cause a loss of one degree of freedom. In addition there are methods simpler for numerical solution then Euler angles, like quaternions (Farrell Jay A., 2017) and direction cosines (Nakath David \& Rachuy, 2017). However they intoduce computational complications like six indemendent differential equations (directions cosines) or transformation matrix for INS navigation is not directaly avaliable (quaternions). The results of the study clearly show that AHRS algorithm considerably accelerates the estimation of Euler angles values, which reduces the time of attitude alignment for integrated systems. On the basis of conducted studies it can be stated that presented AHRS algorithm can be used as a part of INS/GPS integration system.

\section{References}

Cui, X., Mei, C., Qin, Y., Yan, G., \& Fu, Q. (2017). In-motion alignment for low-cost SINS/GPS under random misalignment angles. Journal of Navigation, 1-17. doi: 10.1017/S037346331700039X

Emami, M., \& Taban, M. R. (2017). A customized H-infinity algorithm for underwater navigation system: With experimental evaluation. Ocean Engineering, 130(Supplement C), 611 - 619. Retrieved from http://www.sciencedirect.com/science/article/ pii/S0029801816305996 doi: https://doi.org/10.1016/j.oceaneng.2016.12.011

Farrel, J. A. (2008). Aided navigation GPS with high rate sensors. Mc Graw Hill.

Farrell Jay A., J. W. (2017). GNSS/INS Integration. springer handbook of global navigation satellite systems (S. I. Publishing, Ed.). Springer International Publishing.

Foxlin, E. (2005). Pedestrian tracking with shoe-mounted inertial sensors. IEEE Comput. Graph. Appl(25(6)), 38-46.

Freescale. (2012). Implementing a tilt-compensated eCompass using accelerometer and magnetometer sensors. Freescale Semiconductor Application Note.

Groves, D. (2008). Principles of GNSS, inertial, and multisensory integrated navigation systems. Artech House.

Henriksson, M. (2013). Estimation of heading using magnetometer and GPS.

Li, Z., Wang, J., \& Gao, J. (2016). An enhanced GPS/INS integrated navigation system with GPS observation expansion. Journal of Navigation, 69(5), 1041-1060. doi: $10.1017 /$ S0373463315001083

Nakath David, J. C., \& Rachuy, C. (2017). Rigid body attitude control based on a manifold representation of direction cosine matrices. Journal of Physics: Conference Series., 783(1).

Noureldin, A., Karamat, T., \& J.Georgy. (2013). Fundamentals of inertial navigation, satellitebased positioning and their integration. Springer.

Ozyagcilar, T. (2012). Calibrating an ecompass in the presence of hard and soft-iron interference. Freescale Semiconductor. 
Sabet, M., Daniali, H. M., Fathi, A., \& Alizadeh, E. (2017). Experimental analysis of a low-cost dead reckoning navigation system for a land vehicle using a robust AHRS. Robotics and Autonomous Systems, 95(Supplement C), 37 - 51. Retrieved from http://www.sciencedirect.com/science/article/pii/ S0921889016307497 doi: https://doi.org/10.1016/j.robot.2017.05.010

Sasani, J. A.-S. A. R., S.and Asgari. (2016, Jan 01). Improving MEMS-IMU/GPS integrated systems for land vehicle navigation applications. GPS Solutions, 20(1), 89-100. Retrieved from https://doi.org/10.1007/s10291-015-0471-3 doi: 10.1007/s10291015-0471-3

Simončič, S., Klobčar, D., \& Podržaj, P. (2015). Kalman filter based initial guess estimation for digital image correlation. Optics and Lasers in Engineering, 73(Supplement C), 80 - 88. Retrieved from http://www. sciencedirect.com/science/article/pii/ S0143816615000330 doi: https://doi.org/10.1016/j.optlaseng.2015.03.001

Tang Daquan, J. C. G., Yongkang Jiao. (2016). On automatic landing system for carrier plane based on integration of INS, GPS and vision. In Navigation and control conference (cgncc).

Tomaszewski, D., Rapińki, J., \& Śmieja, M. (2015). Projekt oraz pierwsze testy zitegrowanej platformy ewaluacyjnej GPS/INS. Logistyka(3), 46-49.

Tomaszewski, D., Rapiński, J., \& Śmieja, M. (2015). Analysis of the noise parameters and attitude alignment accuracy of INS conducted with the use of MEMS - based integrated navigation system. Acta Geodynamica et Geomaterialia, 2(12), 197-208.

Wang, L., Zhang, Z., \& Sun, P. (2015). Sun quaternion-based kalman filter for AHRS using an adaptive-step gradient descent algorithm. International Journal of Advanced Robotic Systems(12).

Woodman, O. (2007). An introduction to inertial navigation (Tech. Rep. No. 696). Cambridge.

Yadav, N., \& Bleakley, C. (2014). Accurate orientation estimation using AHRS under conditions of magnetic distortion. Sensors(14), 20008-20024.

Yang Ling, e. a. (2016). Seamless pedestrian navigation augmented by walk status detection and context features. In Ubiquitous positioning, indoor navigation and location based services (upinlbs).

\footnotetext{
Authors: Dariusz Tomaszewski ${ }^{1}$ dariusz.tomaszewski@uwm.edu .pl Jacek Rapiński ${ }^{1}$ jacek.rapinski@uwm .edu.pl Renata Pelc-Mieczkowska² renata.pelc@uwm.edu.pl 1 Institute of Geodesy, The Faculty of Geodesy, Geospatial and Civil Engineering, University of Warmia and Mazury in Olsztyn Oczapowskiego St. 1, 10-719, Olsztyn, Poland 2Institute of Geoinformation and Cartography The Faculty of Geodesy, Geospatial and Civil Engineering, University of Warmia and Mazury in Olsztyn Oczapowskiego St. 1, 10-719, Olsztyn, Poland
} 